Revista Brasileira de Agricultura Irrigada v.14, nº.1, p. 3865 - 3874, 2020

ISSN 1982-7679 (On-line)

Fortaleza, CE, INOVAGRI - http://www.inovagri.org.br

DOI: $10.7127 /$ rbai.v14n1001100

Protocolo 1100.20 - 15/07/2019 Aprovado em 12/05/2020

\title{
UNIFORMIDADE DE DISTRIBUIÇÃO DE ÁGUA E POTÁSSIO UTILIZANDO DIFERENTES TAXAS DE INJEÇÃO DA CALDA FERTILIZANTE
}

\begin{abstract}
Amadeus Mozarth Gomes Rodrigues ${ }^{1}$, Carlos Newdmar Vieira Fernandes ${ }^{2}$,Cristian de França Santos ${ }^{3}$,
\end{abstract} Antonia Euzimar Amorim Sobreira ${ }^{4}$, Jorge Luís de Souza Alves ${ }^{5}$, Joaquim Branco De Oliveira ${ }^{6}$

\section{RESUMO}

Para o sucesso da fertirrigação, é necessário que o sistema de irrigação distribua de forma uniforme a água e nutriente em toda superfície irrigada. Assim, objetivou-se com este estudo avaliar o efeito de diferentes taxas de injeção da calda fertilizante na uniformidade de distribuição de água e potássio em um sistema de irrigação por microaspersão. $\mathrm{O}$ trabalho foi conduzido na área experimental do Instituto Federal de Educação, Ciência e Tecnologia do Ceará - Campus Iguatu, em um sistema de irrigação já instalado em campo. O delineamento experimental utilizado foi de blocos inteiramente casualizados (DIC) com quatro repetições, foram avaliadas as taxas de injeção da calda fertilizante de 40, 60, 80 e $100 \mathrm{~L} \mathrm{~h}^{-1}$, além da operação do sistema sem fertirrigação. A avaliação da uniformidade foi por meio do Coeficiente de Uniformidade de Christiansen (CUC), de Distribuição (CUD) e Estatístico (CUE) para a água de irrigação e do potássio. As análises estatísticas foram realizadas no software ASSISTAT 7.7 e o Microsoft Office Excel 2010. Os coeficientes CUC, CUD e CUE da água de irrigação foram influenciados pelas diferentes taxas de injeção da calda fertilizante. Visando o melhor desempenho do sistema no processo de fertirrigação o produtor poderá optar pela taxa de injeção de $40 \mathrm{~L} \mathrm{~h}^{-1}$. Quanto a uniformidade da concentração e da quantidade de potássio aplicada por emissor não foi influenciada pelas diferentes taxas de injeção avaliadas, ficando a critério do operador do sistema de irrigação utilizar aquela que lhe for mais conveniente.

Palavras-chave: Quimigação, manejo da fertirrigação, coeficiente de uniformidade

\section{WATER AND POTASSIUM DISTRIBUTION UNIFORMITY USING DIFFERENT INJECTION RATES OF THE FERTILIZER}

\footnotetext{
ABSTRACT

${ }^{1}$ Tecnólogo em Irrigação e Drenagem, Discente do Programa de Pós-Graduação em Engenharia Agrícola da UFC, Fortaleza - CE, E-mail: mozarth400@homail.com

${ }^{2}$ Doutor em Engenharia Agrícola. Instituto Federal do Ceará - IFCE. E-mail: newdmar@gmail.com

${ }^{3}$ Tecnólogo em Irrigação e Drenagem, Instituto Federal do Ceará - IFCE. E-mail: fazenda.terraprometida@hotmail.com ${ }^{4}$ Graduanda em Tecnólogo em Irrigação e Drenagem. Instituto Federal do Ceará - IFCE. E-mail: amorim.euzimar@hotmail.com

${ }^{5}$ Graduando em Tecnólogo em Irrigação e Drenagem. Instituto Federal do Ceará - IFCE. E-mail: jgluis27@gmail.com

${ }^{6}$ Doutor em Fitotecnia. Instituto Federal do Ceará - IFCE. E-mail: joaquimbrancodeoliveira@gmail.com
} 
For fertigation success, the irrigation system must distribute water and nutrient evenly on all irrigated surfaces. Thus, the aim of this study was to evaluate the effect of different rates of injection of the fertilizer syrup on the uniformity of water and potassium distribution in a micro sprinkler irrigation system. The work was carried out in the experimental area of the Federal Institute of Education, Science and Technology of Ceará - Campus Iguatu, in an irrigation system already installed in the field. The experimental design was a randomized complete block design (DIC) with four replicates. The injection rates of 40,60, 80 and $100 \mathrm{~L} \mathrm{~h}^{-1}$ fertilizer syringes were evaluated, besides the operation of the system without fertigation. The uniformity evaluation was based on the Christiansen Uniformity Coefficient (CUC), Distribution (CUD) and Statistics (CUE) for irrigation and potassium water. Statistical analyzes were performed using the ASSISTAT 7.7 software and Microsoft Office Excel 2010. The CUC, CUD and CUE coefficients of irrigation water were influenced by the different injection rates of the fertilizer solution. Aiming at the better performance of the system in the fertigation process, the producer may opt for the injection rate of $40 \mathrm{~L} \mathrm{~h}^{-1}$. As for the uniformity of concentration and amount of potassium applied per emitter, it was not influenced by the different injection rates evaluated, leaving the irrigation system operator to use the one that is most convenient.

Keywords: Quimigation, fertigation management, uniformity coeficiente.

\section{INTRODUÇÃO}

A fertirrigação consiste na aplicação de fertilizante em conjunto com água utilizada na irrigação, visando fornecer as quantidades de nutrientes necessárias à cultura naquele momento. O emprego dessa técnica tem possibilitado a otimização do uso de insumos, tanto em aspectos relacionados à produtividade quanto à qualidade final dos produtos obtidos, sendo mais comum sua adoção em culturas irrigadas por sistemas localizados (OLIVEIRA; VILLAS BÔAS, 2008).

A fertirrigação é a prática agrícola que tem permitido aumento de produtividade na agricultura, inclusive viabilizando a produção de frutas e de hortaliças em regiões com baixa disponibilidade hídrica como no Nordeste Brasileiro e, até mesmo, em solos arenosos e de pouca fertilidade. Apesar de ser uma tecnologia incorporada aos diversos sistemas produtivos de fruteiras e hortaliças, o manejo inadequado pode acarreta um grande desperdício de água e insumos, o que pode levar ao aumento da salinidade do solo (OLIVEIRA et al., 2013; SOUSA et al., 2011). Portanto, a fertirrigação deve ser gerenciada para manter uma concentração iônica adequada na solução do solo (NUNES JUNIOR, et al., 2017). Diversos nutrientes são aplicados através da fertirrigação, o potássio é um que se enquadra perfeitamente à fertirrigação, por apresentar elevada mobilidade no solo e a alta solubilidade em água.

O potássio é um macronutriente essencial para as plantas, atuando em diversas funções, entre elas, no transporte de síntese de proteínas de solutos e ativação enzimática. O déficit desse nutriente afeta de forma direta o metabolismo da planta, com consequências negativas sobre a qualidade nutricional do fruto, estabilidade mecânica e resistência a pragas e patógenos. Em excesso o potássio também prejudicar a cultura pela salinização do solo (ARIENZO et al., 2009; ARMENGAUD et al., 2009).

As fontes potássicas mais utilizadas na prática da fertirrigação são: o potássio branco $(\mathrm{KCl} \mathrm{b})$ e vermelho $(\mathrm{KCl} \mathrm{v})$, o sulfato de potássio $\left(\mathrm{K}_{2} \mathrm{SO}_{4}\right)$ e o nitrato de potássio $\left(\mathrm{KNO}_{3}\right)$ (CAMARGO, 2010). O uso desses fertilizantes requer atenção redobrada, principalmente em solos arenosos, por apresentarem elevadas perdas por lixiviação e alto índice salino. Como alternativa para minimizar as perdas da adubação potássica é o parcelamento da fertirrigação (ALBUQUERQUE et al. 2013). Para alcançar o sucesso na aplicação de fertilizante via água de irrigação é necessário que o sistema de irrigação apresente uma boa uniformidade de distribuição de água do sistema, pois a homogeneidade na aplicação de água é um requisito fundamental à prática da fertirrigação. 
Para Santos et al. (2003) a uniformidade de distribuição de água influência diretamente o manejo, a qualidade, a eficiência e o custo da irrigação, assim como o desempenho da cultura no campo.

O mesmo a firma que uma baixa uniformidade de distribuição de água resulta em um crescimento desuniforme dentro do cultivo, devido determinadas plantas receberem mais água e adubo do que outras.

Diante do exposto, objetivou-se nesse estudo, avaliar a uniformidade de distribuição de potássio utilizando diferentes taxas de injeção da calda fertilizante em condições de campo através de um sistema de irrigação localizado por microaspersão.

\section{MATERIAL E MÉTODOS}

O experimento foi conduzido no período de julho a agosto de 2016, na área experimental do Instituto Federal de Educação, Ciência e Tecnologia do Ceará - Campus - Iguatu, localizado no município de Iguatu, a $6^{\circ} 21$ ' $34^{\prime}$ ' de latitude sul e $39^{\circ} 17^{\prime} 55^{\prime}$ ' de longitude oeste e a $217,8 \mathrm{~m}$ de altitude. O clima da região é do tipo Bsw'h'-clima quente e semiárido tipo estepe, com temperatura média mensal superior a $18^{\circ} \mathrm{C}$ no mês mais frio (KÖPPEN, 1923).A precipitação anual média histórica no município de Iguatu é de $867 \pm 304$ mm (1932 a 2011), com evapotranspiração potencial média é de $1.988 \mathrm{~mm} \mathrm{ano}^{-1}$, com maiores valores durante os sete meses de junho a dezembro (FERNANDES et al. 2018). O solo da área apresenta textura franco-arenosa na camada de 0 a 0,2 m (Tabela 1).

Tabela 1. Valores da análise física do solo da área experimental, na camada de 0 a 0,2 m. IFCE, Campus Iguatu.

\begin{tabular}{|c|c|}
\hline Características & $\begin{array}{c}\text { Profundidade (m) } \\
0 \text { a } 0,2\end{array}$ \\
\hline Areia grossa $\left(\mathrm{g} \mathrm{kg}^{-1}\right)$ & 388 \\
\hline Areia fina $\left(\mathrm{g} \mathrm{kg}^{-1}\right)$ & 354 \\
\hline Silte $\left(\mathrm{g} \mathrm{kg}^{-1}\right)$ & 204 \\
\hline Argila $\left(\mathrm{g} \mathrm{kg}^{-1}\right)$ & 54 \\
\hline Classe Textural & Franco - Arenosa \\
\hline Massa específica do solo $\left(\mathrm{g} \mathrm{cm}^{3}\right)$ & 1,5 \\
\hline$* \operatorname{CEes}\left(\mathrm{dS} \mathrm{m}^{-1}\right)$ & 0,4 \\
\hline
\end{tabular}

*CEes = condutividade elétrica do extrato de saturação.

O sistema de irrigação do tipo microaspersão está instalado no campo em uma área de 0,15 hectares, sendo utilizado para irrigação de fruteiras. A linha principal era de PVC com diâmetro nominal de $50 \mathrm{~mm}$, com uma linha de derivação, também de PVC, com diâmetro nominal de $50 \mathrm{~mm}$. Ao longo da linha de derivação, havia 7 linhas laterais, espaçadas entre si em $4 \mathrm{~m}$, compostas por tubos de polietileno de diâmetro nominal de $16 \mathrm{~mm}$, com emissores distanciados entre si em 4,2 m, fornecendo uma vazão individual de $56 \mathrm{~L} \mathrm{~h}^{-1}$, à uma pressão nominal de 20 mca. Para a realização da injeção de fertilizantes, foi instalado, junto ao cabeçal de controle, um sistema injetor do tipo Venturi (Vicamp) de 3/4", com taxa de injeção variando de 1 a $120 \mathrm{~L}$ $\mathrm{h}^{-1}$, auxiliado por uma bomba centrífuga (Inapi) de $1 / 3 \mathrm{cv}$.

Segundo informações levantadas com o operador do sistema, a irrigação era feita de forma diária com um tempo de duas horas, 
também era feita de maneira aleatória a lavagem do sistema. Entretanto, a proposta do experimento era desenvolver a pesquisa em condições reais de campo, analisando a realidade e respeitando sua rotina $\mathrm{e}$ modificando o mínimo possível suas condições de trabalho.

Foi avaliada a uniformidade de distribuição da água e do potássio, esse de acordo com as taxas de injeção da calda fertilizantes. Em todas as taxas avaliadas, foi utilizado a mesma fonte de potássio (Cloreto de potássio branco) e quantidade $\left(1 \mathrm{~kg} \mathrm{ha}^{-1}\right)$, diluído em $10 \mathrm{~L}$ de água, para que a concentração do nutriente não influenciasse nas avaliações. Anterior as avaliações, foi realizada o teste de distribuição da água de irrigação sem a presença de fertilizantes, a fim de se comparar o coeficiente de uniformidade da água de irrigação com os coeficientes de uniformidade do nutriente.

Para avaliar a uniformidade de distribuição do potássio na área, foi utilizada a metodologia de Keller e Karmeli (1975), sendo selecionados 16 pontos de coleta. As linhas laterais selecionadas para a avaliação foram: a primeira, a um terço, a dois terços e a última linha lateral; e mesmo critério foi adotado para a escolha dos microaspersores avaliados (o primeiro, a um terço, a dois terços e o último) ao longo de cada linha lateral selecionada.

$\mathrm{O}$ delineamento experimental do tipo inteiramente casualidade (DIC), com quatro tratamentos e quatro repetições, totalizando 64 amostras coletadas ao final de cada tratamento.
Os tratamentos consistiram na avaliação de diferentes taxas de injeção da calda fertilizante, sendo 40, 60, 80 e $100 \mathrm{~L} \mathrm{~h}^{-1}$.

Para iniciar a coleta de dados do experimento, foi realizada a calibração do injetor Venturi para ajustar a taxas a serem avaliadas. A determinação do tempo de fertirrigação para coleta de água nos pontos pré-determinados, procedeu-se da seguinte forma: primeiramente foi medida a condutividade elétrica da água de irrigação sem adição de fertilizante com auxílio de um condutivímetro portátil, logo após injetava-se a calda fertilizante no sistema e no momento em que a condutividade elétrica do primeiro microaspersor da área experimental apresentava-se superior à condutividade elétrica da água medida anteriormente, iniciava a coleta da água, pois indicava que o fertilizante havia chegado à área. $\mathrm{O}$ final do tempo de coleta era determinado quando a condutividade elétrica da água coletada no último. Por fim, o sistema de irrigação era desligado e retiravamse os coletores, para em seguida medir-se o volume da solução utilizando provetas graduadas em $\mathrm{mL}$, retirando-se uma amostra em recipientes de plástico de $20 \mathrm{ml}$, devidamente identificados para posterior análise.

Outros aspectos importantes para avaliação do sistema foram avaliados, como a pressão de serviço, diferença de pressão, volume de calda, tempo de injeção e tempo de coleta obtido para os diferentes tratamentos avaliados (Tabela 2).

Tabela 2. Valores médios de pressão, diferença de pressão, volume de calda, tempo de injeção e tempo de coleta para os diferentes tratamentos avaliados. IFCE, Campus Iguatu.

\begin{tabular}{cccccc}
\hline Tratamento & $\begin{array}{c}\text { Ps } \\
(\mathrm{mca})\end{array}$ & $\begin{array}{c}\Delta \mathrm{P} \\
(\mathrm{mca})\end{array}$ & $\begin{array}{c}\text { Volume da } \\
\text { calda (L) }\end{array}$ & $\begin{array}{c}\text { Tempo de } \\
\text { injeção (min) }\end{array}$ & $\begin{array}{c}\text { Tempo de coleta } \\
(\mathrm{min})\end{array}$ \\
\hline $\begin{array}{c}\text { Sem } \\
\text { fertirrigação }\end{array}$ & 20 & - & - & - & - \\
$40 \mathrm{~L} \mathrm{~h}^{-1}$ & 15 & 25 & 10 & 15 & 27 \\
$60 \mathrm{~L} \mathrm{~h}^{-1}$ & 13 & 27 & 10 & 10 & 21 \\
$80 \mathrm{~L} \mathrm{~h}^{-1}$ & 10 & 31 & 10 & 7,5 & 18 \\
$100 \mathrm{~L} \mathrm{~h}^{-1}$ & 4 & 39 & 10 & 6 & 17 \\
\hline
\end{tabular}

Ps - pressão de serviço; $\Delta \mathrm{P}$ - diferença de pressão.

Feito todas as anotações, as amostras foram levadas ao Laboratório de Análise de
Água, Solo e Tecidos Vegetais - LABAS, do Instituto Federal de Educação, Ciência e 


\section{UNIFORMIDADE DE DISTRIBUIÇÃO DE ÁGUA E POTÁSSIO UTILIZANDO \\ DIFERENTES TAXAS DE INJEÇÃO DA CALDA FERTILIZANTE}

Tecnologia do Ceará, Campus Iguatu, para a quantificar o potássio de todas as amostras, através de um espectrofotômetro de chamas. Para o cálculo da uniformidade de distribuição da água e do nutriente, foram utilizadas as equações 1,2 e 3 . Para análise da uniformidade da concentração do potássio substituiu-se as vazões em $\mathrm{L} \mathrm{h}{ }^{-1}$, pela concentração das amostras analisadas em $\mathrm{g} \mathrm{L}^{-1}$, enquanto, para a uniformidade de distribuição da quantidade do nutriente por emissor substituiu-se pela quantidade obtida na equação 4.

$$
\begin{gathered}
C U C=100 \cdot\left(1-\frac{\sum_{i=1}^{n}\left(\left|q_{i}-\bar{q}\right|\right)}{n \cdot \bar{q}}\right) \\
C U D=100 \cdot\left(\frac{q_{25 \%}}{q}\right) \\
C U E=100 \cdot\left(1-\frac{S_{q}}{-}\right) \\
\text { Quant. }=\text { Vol } * C_{n}
\end{gathered}
$$

Em que: CUC - coeficiente de uniformidade de Christiansen (\%); CUD coeficiente de uniformidade de distribuição (\%); CUE - é o coeficiente de uniformidade estatístico (\%); Quant. - Quantidade de nutriente coletada (g); qi - vazão de cada emissor $\left(\mathrm{L} \mathrm{h}^{-1}\right) ; \overline{\mathrm{q}}$ - vazão média dos emissores $\left(\mathrm{L} \mathrm{h}^{-1}\right) ; \mathrm{n}$ - número de emissores; $\mathrm{q} 25 \%$ média de $25 \%$ das menores vazões observadas $\left(\mathrm{L} \mathrm{h}^{-1}\right) ; \mathrm{Sq}$ - é o desvio padrão de vazão do emissor; Vol - é o volume coletado em cada emissor; $\mathrm{Cn}$ - concentração do nutriente no emissor $\left(\mathrm{g} \mathrm{L}^{-1}\right)$. A interpretação dos valores dos coeficientes de uniformidade (CUC, CUE e 


\section{RESULTADOS E DISCUSSÃO}

Pelos dados apresentados na Tabela 2, se observa que à medida em que houve aumento na taxa de injeção da calda fertilizante ocorreu uma redução na pressão de serviço do sistema de irrigação, em virtude da necessidade de um maior diferencial de pressão capaz de proporcionar a taxa requerida, mesmo utilizando uma bomba auxiliar. Tal fato corrobora com a conclusão de Ferreira et al. (1996) de que a vazão de sucção aumenta com o aumento do diferencial de pressão. Na Tabela 4 observa-se o resumo da análise de variância para o Coeficiente de Uniformidade de Christiansen (CUC), o Coeficiente de Uniformidade de Distribuição (CUD) e Coeficiente de Uniformidade Estatístico (CUE) da água de irrigação, para os tratamentos avaliados.

Verifica-se que todos os coeficientes de uniformidade apresentaram diferença significativa, sendo o CUC e o CUE a 5\% $(\mathrm{P}<0,05)$ enquanto o CUD a $1 \%(\mathrm{P}<0,01)$.

Tabela 4. Análise de variância do CUC, CUD e CUE da água de irrigação em função das taxas de injeção avaliadas. IFCE, Campus Iguatu.

\begin{tabular}{ccccc}
\hline \multirow{2}{*}{ FV } & \multirow{2}{*}{ GL } & \multicolumn{3}{c}{ Quadrado médio } \\
\cline { 3 - 5 } & 4 & $1,87553^{*}$ & CUD & CUE \\
\hline Tratamentos & 15 & 0,60386 & $0,51801^{* *}$ & $8,88930^{*}$ \\
Resíduo & 19 & - & - & 0,53738 \\
\hline Total & - & 0,85 & 0,85 & - \\
\hline CV $(\%)$ & FV & Fator de Variação; GL - Grau de liberdade; CV - Coeficiente de variação; ** significativo a 1\% pelo teste F; * significativo a 5\% pelo teste F.
\end{tabular}

Os valores médios do CUC, CUD e CUE da água de irrigação em função das taxas de injeção avaliadas estão apresentados na Tabela 5. Pelo teste de Tukey o CUC não apresentou diferença significativa entre os tratamentos avaliados. Com relação ao CUD, os tratamentos sem fertirrigação e taxa de $40 \mathrm{~L} \mathrm{~h}^{-1}$ apresentaram os maiores valores médios.

Já para o CUE o tratamento sem fertirrigação apresentou o melhor valor, sendo a taxa de injeção de $40 \mathrm{~L} \mathrm{~h}^{-1}$ estatisticamente igual.

Tabela 5. Teste de Tukey para os valores médios do CUC, CUD e CUE da água de irrigação em função das taxas de injeção avaliadas. IFCE, Campus Iguatu.

\begin{tabular}{cccc}
\hline \multirow{2}{*}{ Tratamento } & \multicolumn{3}{c}{ Coeficiente de uniformidade } \\
\cline { 2 - 4 } & CUC $(\%)$ & CUD $(\%)$ & CUE $(\%)$ \\
\hline Sem fertirrigação & $91,92 \mathrm{a}$ & $83,72 \mathrm{a}$ & $88,10 \mathrm{a}$ \\
$40 \mathrm{~L} \mathrm{~h}^{-1}$ & $91,65 \mathrm{a}$ & $83,18 \mathrm{a}$ & $86,57 \mathrm{ab}$ \\
$60 \mathrm{~L} \mathrm{~h}^{-1}$ & $91,11 \mathrm{a}$ & $82,26 \mathrm{ab}$ & $85,54 \mathrm{bc}$ \\
$80 \mathrm{~L} \mathrm{~h}^{-1}$ & $90,52 \mathrm{a}$ & $80,92 \mathrm{~b}$ & $84,11 \mathrm{c}$ \\
$100 \mathrm{~L} \mathrm{~h}^{-1}$ & $90,35 \mathrm{a}$ & $81,39 \mathrm{~b}$ & $85,38 \mathrm{bc}$ \\
\hline Média & 91,11 & 82,30 & 85,84 \\
\hline
\end{tabular}

Os valores de todos os Coeficientes de Uniformidade de Christiansen (CUC) foram apontados como excelentes de acordo com a classificação proposta por Mantovani (2001) (Tabela 3). Isto evidenciando que o sistema de irrigação foi hidraulicamente bem dimensionado e está funcionando apropriadamente, sem risco de comprometer a produção pela desuniformidade de distribuição da água ou fertilizante.
O valor médio do CUC encontrado nesse trabalho foi superior ao observado por Azevedo et al. (2014), que obteve resultado inferior, com o CUC de 85,23\% para um sistema de irrigação por gotejamento. Feitosa et al. (2012) obtiveram maiores valores de CUC $(97,43 \%)$, ao avaliar a uniformidade de distribuição de um sistema de irrigação por microasperção, município de Missão Velha-CE. Para Bernardo et al. (2006), sistemas de irrigação com valores 


\section{UNIFORMIDADE DE DISTRIBUIÇÃO DE ÁGUA E POTÁSSIO UTILIZANDO DIFERENTES TAXAS DE INJEÇÃO DA CALDA FERTILIZANTE}

de CUC superiores a $90 \%$ são recomendado para culturas de alto rendimento econômico, desta forma o sistema avaliado pode ser utilizado para tais culturas, já que o valor do CUC em nenhum tratamento ficou abaixo de $90 \%$.

O CUD tem papel importante, uma vez que esse coeficiente compara a média de $25 \%$ das observações de valores mais baixos com a média total, sendo por isso, o mais utilizado na avaliação de sistemas de irrigação localizada, por possibilitar uma medida precisa (LÓPEZ et al., 1992). Essa maior precisão proporcionada pelo CUD contribuiu para a melhor observação das diferenças entre os tratamentos avaliados, uma vez que, para esse coeficiente o teste de médias demonstrou diferença entres os tratamentos. Desta forma fica claro que a taxa de injeção de $40 \mathrm{~L} \mathrm{~h}^{-1}$ deve ser a escolhida quando se desejar realizar a prática da fertirrigação no referido sistema, a fim de se alcançar uma maior uniformidade na irrigação.

No que diz respeito ao Coeficiente de Uniformidade Estatístico (CUE), estes foram classificados como bons, em todos os tratamentos, de acordo com Tabela 3. No entanto, ressalta-se que pelo teste de comparação entres as médias, a taxa de injeção de $40 \mathrm{~L} \mathrm{~h}^{-1}$ foi o único tratamento que não diferiu estatisticamente da operação do sistema sem fertirrigação, fato que evidencia a afirmação anterior de que a referida taxa de injeção deve ser a escolhida para a realização da fertirrigação no sistema.

Os menores valores de CUD e CUE verificados para as maiores taxas de injeção avaliadas comprometem o desenvolvimento uniforme da cultura, uma vez que, este é fortemente influenciado pela uniformidade de distribuição de água em áreas irrigadas, onde baixas uniformidades fazem com que determinadas plantas recebam menos água do que outras afetando o seu desenvolvimento (SANTOS et al., 2003). Diversos fatores podem interferir no desempenho deste sistema de irrigação, entre as principais estão a obstrução das mangueiras emissoras, a oscilação de pressão da rede e falhas hidráulicas no dimensionamento e operação (CARMO et al., 2016).

$\mathrm{Na}$ Tabela 6 está apresentado o resumo da análise de variância para o Coeficiente de Uniformidade de Christiansen (CUC), o Coeficiente de Uniformidade de Distribuição (CUD) e Coeficiente de Uniformidade Estatístico (CUE) da concentração de potássio, para os tratamentos avaliados. Observa-se que todos os coeficientes de uniformidade não apresentaram diferença estatística em nível de $1 \%$ e $5 \%$ de significância pelo teste $\mathrm{F}$.

Tabela 6. Análise de variância do CUC, CUD e CUE da concentração de potássio em função das taxas de injeção avaliadas. IFCE, Campus Iguatu.

\begin{tabular}{ccccc}
\hline \multirow{2}{*}{ FV } & \multirow{2}{*}{ GL } & CUC & Quadrado médio \\
\cline { 3 - 5 } & 3 & $1,02967^{\mathrm{ns}}$ & $2,82376^{\mathrm{ns}}$ & CUE \\
\hline Tratamentos & 12 & 2,64465 & 9,39197 & $1,07148^{\mathrm{ns}}$ \\
Resíduo & 15 & - & - & 5,09330 \\
\hline Total & - & 1,80 & 3,64 & - \\
\hline CV $(\%)$ & FV - Fator de Variação; GL - Grau de liberdade; CV - Coeficiente de variação; ns - não significativo pelo teste F.
\end{tabular}

Os valores médios do CUC, CUD e CUE da concentração de potássio em função das taxas de injeção avaliadas estão apresentados na Tabela 7. De acordo com a classificação proposta por Mantovani (2001), os valores de
CUC e CUD foram excelentes e os valores para o CUE foram bons, demonstrando que o sistema de irrigação avaliado é eficiente na distribuição de nutriente. 
Tabela 7. Valores médios do CUC, CUD e CUE da concentração de potássio em função das taxas de injeção avaliadas. IFCE, Campus Iguatu.

\begin{tabular}{cccc}
\hline \multirow{2}{*}{ Tratamento } & \multicolumn{3}{c}{ Coeficiente de uniformidade } \\
\cline { 2 - 4 } & CUC $(\%)$ & CUD $(\%)$ & CUE (\%) \\
\hline $40 \mathrm{~L} \mathrm{~h}^{-1}$ & 90,18 & 84,51 & 87,55 \\
$60 \mathrm{~L} \mathrm{~h}^{-1}$ & 89,80 & 84,09 & 86,67 \\
$80 \mathrm{~L} \mathrm{~h}^{-1}$ & 90,55 & 83,09 & 87,44 \\
$100 \mathrm{~L} \mathrm{~h}^{-1}$ & 90,98 & 85,08 & 87,90 \\
\hline Média & 90,38 & 84,20 & 82,31 \\
\hline
\end{tabular}

A não significância para os dados avaliados, pode estar associado a uma baixa amplitude de variação da taxa de injeção (40 a $100 \mathrm{~L} \mathrm{~h}^{-1}$ ), amplitude essa que foi limitada pelo equipamento (venturi) utilizado, uma vez que o mesmo apresentava $100 \mathrm{~L} \mathrm{~h}^{-1}$ como limite máximo de injeção.

Os elevados valores dos coeficientes avaliados e a inexistência de diferença estatística entre os tratamentos indicam uma correta solubilidade e homogeneidade do fertilizante e uma ótima distribuição pelo sistema de irrigação, nesse caso, o produtor poderá optar por aquela que lhe for mais conveniente. Outro ponto a destacar é o tempo de aplicação do fertilizante via água de irrigação.

Oliveira e Villas Bôas (2008) ressaltam que quanto maior o tempo de funcionamento de sistemas de irrigação, na operação de fertirrigação, melhor a uniformidade de distribuição do fertilizante. Corroborando com esse fato, Cavalcante Júnior (2013) avaliando a uniformidade de distribuição do $\mathrm{K}_{2} \mathrm{O}$ em função de diferentes injetores, obteve os seguintes tempos de aplicação para os injetores avaliados: 17,53 minutos (Venturi A, com diâmetro de $25 \mathrm{~mm}$ ), 14,34 minutos (Venturi B, com diâmetro de $25 \mathrm{~mm}$ e dispositivo de controle de taxa de injeção), 1,28 minutos (bomba centrífuga) e 1,49 minutos (tanque pressurizado). Segundo o autor, o maior tempo de injeção no caso 17,53 minutos, resultou em maior CUC 94,66\% da distribuição de potássio.

$\mathrm{Na}$ Tabela 8 pode ser observado o resumo da análise de variância do CUC, CUD e CUE da quantidade de potássio por emissor em função das quatro taxas de injeção avaliadas. Verifica-se que nenhum dos coeficientes de uniformidade avaliados apresentou diferença significativa.

Na Tabela 9, estão apresentados os valores médios para os coeficientes de uniformidade CUC, CUD e CUE da quantidade de potássio por emissor em função dos tratamentos aplicados.

Tabela 8. Análise de variância do CUC, CUD e CUE da quantidade de potássio por emissor em função das taxas de injeção avaliadas. IFCE, Campus Iguatu.

\begin{tabular}{ccccc}
\hline \multirow{2}{*}{ FV } & \multirow{2}{*}{ GL } & \multicolumn{3}{c}{ Quadrado médio } \\
\cline { 3 - 5 } & 3 & $3,34541^{\text {ns }}$ & CUD & CUE \\
\hline Tratamentos & 12 & 4,77987 & $3,09884^{\text {ns }}$ & $3,49248^{\text {ns }}$ \\
Resíduo & 15 & - & - & 1,95014 \\
\hline Total & - & 2,54 & 2,35 & - \\
\hline CV $(\%)$ & FV - Fator de Variação; GL - Grau de liberdade; CV - Coeficiente de variaç̃o; ns - não significativo pelo teste F.
\end{tabular}




\section{UNIFORMIDADE DE DISTRIBUIÇÃO DE ÁGUA E POTÁSSIO UTILIZANDO DIFERENTES TAXAS DE INJEÇÃO DA CALDA FERTILIZANTE}

Tabela 9. Valores médios do CUC, CUD e CUE da quantidade de potássio por emissor. IFCE, Campus Iguatu.

\begin{tabular}{cccc}
\hline \multirow{2}{*}{ Tratamento } & \multicolumn{3}{c}{ Coeficiente de uniformidade } \\
\cline { 2 - 4 } & CUC $(\%)$ & CUD $(\%)$ & CUE $(\%)$ \\
\hline $40 \mathrm{~L} \mathrm{~h}^{-1}$ & 84,80 & 78,56 & 83,59 \\
$60 \mathrm{~L} \mathrm{~h}^{-1}$ & 86,71 & 76,66 & 81,55 \\
$80 \mathrm{~L} \mathrm{~h}^{-1}$ & 86,43 & 75,08 & 81,69 \\
$100 \mathrm{~L} \mathrm{~h}^{-1}$ & 86,69 & 76,89 & 82,40 \\
\hline Média & 86,16 & 76,80 & 82,31 \\
\hline
\end{tabular}

Os valores de CUC, CUE e CUE para as respectivas taxas avaliadas em relação a quantidade de potássio emitido por emissor é considerada "bom" (MANTOVANI, 2001). Para Bomfim et al. (2014) quanto mais próximo a injeção de fertilizante for das linhas laterais, maior é a quantidade de nutriente fornecido pelo sistema.

Outro fator que pode ser associado a não variação na quantidade de nutriente e uma maior ação do movimento de difusão nas extremidades das linhas laterais (OLIVEIRA; VILLAS BÔAS, 2008).

Pelos resultados apresentados nas Tabelas 8 e 9 pode-se constatar que apesar da influência das taxas de injeção na uniformidade de distribuição de água do sistema de irrigação (Tabelas 4 e 5), essa não foi o bastante para provocar um efeito significativo na uniformidade de distribuição da quantidade de nutriente aplicada.

\section{CONCLUSÃO}

Os Coeficiente de Uniformidade de Christiansen (CUC), o Coeficiente de Uniformidade de Distribuição (CUD) e Coeficiente de Uniformidade Estatístico (CUE) da água de irrigação foram influenciados pelas diferentes taxas de injeção da calda fertilizante.

O produtor poderá optar pela taxa de injeção de $40 \mathrm{~L}^{-1}$ para a prática da fertirrigação nas condições em que foi avaliado o sistema, pois foi o único tratamento que não diferiu estatisticamente da operação do sistema sem fertirrigação.

A uniformidade da concentração e da quantidade de potássio aplicada por emissor não foi influenciada pelas diferentes taxas de injeção avaliadas, ficando a critério do operador do sistema de irrigação utilizar aquela que lhe for mais conveniente.

\section{REFERÊNCIAS BIBLIOGRÁFICAS}

ALBUQUERQUE, A. H. P.; VIANA, T. V. A.; MARINHO, A. B.; SOUSA, G. G.; AZEVEDO, B. M. Irrigação e fertirrigação potássica na cultura da videira em condições semiáridas. Pesquisa Agropecuária Tropical, v. 43, n. 3, p. 315-321, 2013.

ARIENZO, M.; CHRISTEN, E. W.; QUAYLE, W.; KUMAR, A. A review of the fate of potassium in the soil-plant system after land application of wastewaters. Journal of Hazardous Materials, v. 164, n. 2-3, p. 415422, 2009.

ARMENGAUD, $\quad$ P.; SULPICE, R.; MILLER, A. J.; STITT, M.; AMTMANN, A.; GIBON, Y. Multilevel analysis of primary metabolism provides new insights into the role of potassium nutrition for glycolysis and nitrogen assimilation in arabidopsis roots. Plant Physiology, v. 150, n. 2, p. 772-785, 2009.

AZEVEDO, B. M.; FERNANDES, C. $\quad$ N. V.; CAMARGO, D. C.; GARCÍA, A. C.; VIANA, T. V. A.; CAMPÊLO, A. R. Fertirrigação potássica do cajueiro com diferentes fontes e formas de diluição do cloreto de potássio. Irriga, v. 19, n. 2, p. 325-332, 2014. 
BERNARDO, S.; SOARES, A. A.; MANTOVANI, E. C. Manual de Irrigação. 8. ed. Viçosa, MG: UFV, 2006. 625 p.

BOMFIM, G. V. D.; MANZANO, J.; AZEVEDO, B. M. D.; VASCONCELOS, D. V.; VIANA, T. V. D. A. Potassium distribution in drip irrigation with fertigation for different injection distances in the main line. Engenharia Agrícola, v. 34, n. 6, p. 11501161, 2014.

CAMARGO, D. C. Uniformidade de distribuição de potássio utilizando diferentes fontes e concentrações na calda de fertirrigação em condições de campo. Fortaleza, 2010. 66 f. Dissertação (Mestrado em Engenharia Agrícola) - Universidade Federal do Ceará, UFC.

CAVALCANTE JÚNIOR, J. A. H. Frequência de fertirrigação potássica no meloeiro e distribuição de $\mathrm{K}_{2} \mathrm{O}$ com diferentes injetores e idade de utilização do sistema de irrigação. Fortaleza, 2013. 82 f. Tese (Doutorado em Engenharia Agrícola) Universidade Federal do Ceará, UFC.

FERNANDES, C. N. D.; RODRIGUES, A. M. G.; VIANA, T. V. A.; FERNANDES, C. N. V.; SOBREIRA, A. E. A.; AZEVEDO, B. M. Crescimento do milho verde sob lâminas de irrigação e adubação foliar silicatada. Revista Brasileira de Agricultura Irrigada, v.12, $\mathrm{n}^{\mathrm{o}} .4$ (edição especial V WINOTEC), p. 2789 2798, 2018.

FERREIRA, J. O. P.; FRIZZONE, J. A.; BOTREL, T. A. Desempenho de um injetor de fertilizantes do tipo venturi para fins de fertirrigação. Revista Scientia Agrícola. v.53, n. 1, p. 105-113. 1996. KELLER, J.; KARMELI, D. Trickle irrigation design parameters. Transactions of the ASAE, v. 17, n.4, p.878-880, 1975.
LÓPEZ, J. R.; ABREU, J. M. H.; REGALADO, A.P.; HERNADEZ, J.F.G. Riego localizado. 2 ed. Madrid: Centro Nacional de Tecnologia de Regadios, 1992. p.217-229.

MANTOVANI, E. C. AVALIA: Programa de Avaliação da Irrigação por Aspersão e Localizada. Viçosa, MG: UFV, 2001

NUNES JÚNIOR, E. S.; MEDEIROS, J. F.; OLIVEIRA, F. A.; LIMA, L. A.; BEZERRA, F. M. S.; ALVES, R. C. Nitrogen and potassium fertigation in bell pepper cultivated in greenhouse using fertigation managements. Revista Brasileira de Engenharia Agrícola e Ambiental, v.31, n.3, p. 186-190, 2017.

OLIVEIRA, M. V. A. M.; VILLAS BÔAS, R. L. Uniformidade de distribuição do potássio e do nitrogênio em sistema de irrigação por gotejamento. Engenharia Agrícola, v. 28, n. 1, p. 95-103, 2008.

OLIVEIRA, F. A.; DUARTE, S. N.; MEDEIROS, J. F; DIAS, N. S; SILVA, R. C. P; LIMA, C. J. G. S. Manejos da fertirrigação e doses de $\mathrm{N}$ e $\mathrm{K}$ no cultivo de pimentão em ambiente protegido. Revista Brasileira de Engenharia Agrícola e Ambiental, v.17, p.1152-1159, 2013.

SANTOS, R. A.; HERNANDEZ, F. B. T.; FERREIRA, E. J. S.; VANZELA, L. S.; LIMA, R. C. Uniformidade de distribuição de água em irrigação por gotejamento em sub-superfície instalado na cultura de pupunheiras (Bactris gasipaes h.b.k.). In: Congresso Brasileiro de Engenharia Agrícola, 32, 2003, Goiânia. Anais... Piracicaba: SBEA, 2003. p. 1-4.

SOUSA, V. F. MAROUELLI, W. A.; COELHO, E. F.; PINTO, J. M.; COELHO FILHO, M. A. Irrigação e fertirrigação em fruteiras e hortaliças. Brasília, DF: Embrapa Informação Tecnológica, 2011. 771 p. 